\title{
Classification of simple weight modules for the Neveu-Schwarz algebra with a finite-dimensional weight space
}

\author{
Xiufu Zhang ${ }^{1}$, Zhangsheng Xia ${ }^{2}$ \\ 1. School of Mathematical Sciences, Xuzhou Normal University, Xuzhou 221116, China \\ 2. School of Sciences, Hubei University for Nationalities, Enshi 445000, China
}

\begin{abstract}
We show that the support of a simple weight module over the NeveuSchwarz algebra, which has an infinite-dimensional weight space, coincides with the weight lattice and that all non-trivial weight spaces of such module are infinite-dimensional. As a corollary we obtain that every simple weight module over the Neveu-Schwarz algebra, having a non-trivial finitedimensional weight space, is a Harish-Chandra module (and hence is either a highest or lowest weight module, or else a module of the intermediate series). This result generalizes a theorem which was originally given on the Virasoro algebra.
\end{abstract}

2000 Mathematics Subject Classification: 17B10, 17B65, 17B68

Keywords: Neveu-Schwarz algebra, weight module, Harish-Chandra module.

\section{Introduction}

It is well known that the Virasoro algebra Vir plays a fundamental role in twodimensional conformal quantum field theory. As the super-generalization, there are two super-Virasoro algebras called the Neveu-Schwarz algebra and the Ramond algebra corresponding to $N=\frac{1}{2}$ and $N=1$ super-conformal field theory respectively. Let $\theta=\frac{1}{2}$ or 0 which corresponds to the Neveu-Schwarz case or the Ramond case respectively. The super-Virasoro algebra $S \operatorname{Vir}(\theta)$ is the Lie superalgebra $S \operatorname{Vir}(\theta)=$ $S V i r_{\overline{0}} \oplus S V i r_{\overline{1}}$, where $S V i r_{\overline{0}}$ has a basis $\left\{L_{n}, c \mid n \in \mathbb{Z}\right\}$ and $S V i r_{\overline{1}}$ has a basis

\footnotetext{
* Supported by the National Natural Science Foundation of China (No. 10931006).

** Email: xfzhang@xznu.edu.cn; xzsw8577@163.com
} 
$\left\{G_{r} \mid r \in \theta+\mathbb{Z}\right\}$, with the commutation relations

$$
\begin{aligned}
& {\left[L_{m}, L_{n}\right]=(n-m) L_{n+m}+\delta_{m+n, 0} \frac{m^{3}-m}{12} c,} \\
& {\left[L_{m}, G_{r}\right]=\left(r-\frac{m}{2}\right) G_{m+r},} \\
& {\left[G_{r}, G_{s}\right]=2 L_{r+s}+\frac{4 r^{2}-1}{12} \delta_{r+s, 0} c} \\
& {\left[S V i r_{\overline{0}}, c\right]=0=\left[S V i r_{\overline{1}}, c\right]}
\end{aligned}
$$

for $m, n \in \mathbb{Z}, r, s \in \theta+\mathbb{Z}$.

The subalgebra $\mathfrak{h}=\mathbb{C} L_{0} \oplus \mathbb{C} c$ is called the Cartan subalgebra of $\operatorname{SVir}(\theta)$. An $\mathfrak{h}$-diagonalizable $S \operatorname{Vir}(\theta)$-module is usually called a weight module. If $M$ is a weight module, then $M$ can be written as a direct sum of its weight spaces, $M=\bigoplus M_{\lambda}$, where $M_{\lambda}=\left\{v \in M \mid L_{0} v=\lambda\left(L_{0}\right) v, c v=\lambda(c) v\right\}$. We call $\left\{\lambda \mid M_{\lambda} \neq 0\right\}$ the support of $M$ and is denoted by $\operatorname{supp}(M)$. A weight module is called Harish-Chandra module if each weight space is finite-dimensional.

In [1], [2], [3], [7], the unitary modules, the Verma modules, the Fock modules and the Harish-Chadra modules over $S \operatorname{Vir}(\theta)$ are studied. In this paper, we try to give the classification of simple weight modules for the Neveu-Schwarz algebra with a finite-dimensional weight space. Our main result generalizes a theorem which was originally given on the Virasoro algebra in [6].

For convenience, we denote the Neveu-Schwarz algebra by $N S$ instead of $S \operatorname{Vir}\left(\frac{1}{2}\right)$ and we define

$$
E_{k}= \begin{cases}L_{k}, & \text { if } k \in \mathbb{Z}, \\ G_{k}, & \text { if } k \in \frac{1}{2}+\mathbb{Z} .\end{cases}
$$

Suppose $M$ is a simple weight $N S$-module, then $c$ acts on $M$ by a scalar and $M$ can be written as a direct sum of its weight spaces, $M=\bigoplus M_{\lambda}$, where $M_{\lambda}=$ $\left\{v \in M \mid L_{0} v=\lambda v\right\}$. Obviously, if $\lambda \in \operatorname{supp}(M)$, then $\operatorname{supp}(M) \subseteq\left\{\lambda+k \mid k \in \frac{\mathbb{Z}}{2}\right\}$, the weight lattice. Two elements $i, j \in\left\{\lambda+k \mid k \in \frac{\mathbb{Z}}{2}\right\}$ are called adjacent if $|i-j|=\frac{1}{2}$, otherwise, unadjacent.

In this paper, our main result is the following theorem:

Theorem 1. Let $M$ be a simple weight $N S$-module. Assume that there exists $\lambda \in \mathbb{C}$ such that $\operatorname{dim} M_{\lambda}=\infty$. Then $\operatorname{supp}(M)=\lambda+\frac{\mathbb{Z}}{2}$ and for each $k \in \frac{\mathbb{Z}}{2}$ we have $\operatorname{dim}\left(M_{\lambda+k}\right)=\infty$.

In [7], Y. Su proved the following result which generalizes a theorem originally given as a conjecture by Kac in [4] on the Virasoro algebra and proved by Mathieu in $[5]$.

Theorem 2. A Harish-Chandra module over $\operatorname{SVir}(\theta)$ is either a highest or lowest weight module, or else a module of the intermediate series.

By Theorem 1 and 2, we get the following corollaries immediately: 
Corrollary 3. Let $M$ be a simple weight $N S$-module. Assume that there exists $\lambda \in$ $\mathbb{C}$ such that $0<\operatorname{dim} M_{\lambda}<\infty$. Then $M$ is a Harish-Chandra module. Consequently, $M$ is either a highest or lowest weight module, or else a module of the intermediate series.

A weight $N S$-module, $M$, is called mixed module if there exist $\lambda \in \mathbb{C}$ and $k \in \frac{\mathbb{Z}}{2}$ such that $\operatorname{dim} M_{\lambda}=\infty$ and $\operatorname{dim} M_{\lambda+k}<\infty$.

Corrollary 4. There are no simple mixed NS-modules.

\section{Proof of the Theorem 1}

Noting that $\left\{G_{-\frac{3}{2}}, G_{-\frac{1}{2}}, G_{\frac{1}{2}}, G_{\frac{3}{2}}\right\}$ is a set of generators of $N S$, we have the following fact immediately:

Principal Fact: Assume that there exists $\mu \in \mathbb{C}$ and $0 \neq v \in M_{\mu}$, such that $G_{\frac{1}{2}} v=G_{\frac{3}{2}} v=0$ or $G_{-\frac{1}{2}} v=G_{-\frac{3}{2}} v=0$. Then $M$ is a Harish-Chandra $N S$-module.

Lemma 1. If there are $\mu, \mu^{\prime} \in\left\{\lambda+k \mid k \in \frac{\mathbb{Z}}{2}\right\}$ such that $\operatorname{dim} M_{\mu}<\infty$ and $\operatorname{dim} M_{\mu^{\prime}}<$ $\infty$, then $\mu, \mu^{\prime}$ are adjacent.

Proof. Suppose that there exist two unadjacent elements in $\left\{\lambda+k \mid k \in \frac{\mathbb{Z}}{2}\right\}$ correspond to finite-dimensional weight spaces or trivial vector spaces in $M$. Without loss of generality, we may assume that $\operatorname{dim} M_{\lambda+\frac{1}{2}}<\infty$ and $\operatorname{dim} M_{\lambda+k}<\infty$, where $k \in\left\{\frac{3}{2}, 2, \frac{5}{2}, 3, \cdots\right\}$. Let $V$ denote the intersection of the kernels of the linear maps:

$$
G_{\frac{1}{2}}: M_{\lambda} \rightarrow M_{\lambda+\frac{1}{2}}
$$

and

$$
E_{k}: M_{\lambda} \rightarrow M_{\lambda+k}
$$

Since $\operatorname{dim} M_{\lambda}=\infty, \operatorname{dim} M_{\lambda+\frac{1}{2}}<\infty$, we know that the kernel $\operatorname{ker} G_{\frac{1}{2}}$ of $G_{\frac{1}{2}}: M_{\lambda} \rightarrow$ $M_{\lambda+\frac{1}{2}}$ is infinite dimensional. Since $\operatorname{dim} M_{\lambda+k}<\infty$, we also have that the kernel of $\left.E_{k}\right|_{k e r G_{\frac{1}{2}}}: \operatorname{ker} G_{\frac{1}{2}} \rightarrow M_{\lambda+k}$ is infinite dimensional. That is $\operatorname{dim} V=\infty$.

Since

$$
\left[G_{\frac{1}{2}}, E_{l}\right]= \begin{cases}2 L_{\frac{1}{2}+l}, & \text { if } l \in \frac{1}{2}+\mathbb{N} \\ \left(\frac{l}{2}-\frac{1}{2}\right) G_{\frac{1}{2}+l}, & \text { if } l \in \mathbb{Z}_{+} \backslash\{1\}\end{cases}
$$

is nonzero, we get that

$$
E_{l} V=0, \forall l=\frac{1}{2}, k, k+\frac{1}{2}, k+1, \cdots
$$

If $k=\frac{3}{2}$ then there exists $0 \neq v \in V$ such that $G_{\frac{3}{2}} v=0$, and $M$ would be a Harish-Chandra module by the Principal Fact, a contradiction. Thus $k>\frac{3}{2}$ and $\operatorname{dim} G_{\frac{3}{2}} V=\infty$. Since $\operatorname{dim} M_{\lambda+\frac{1}{2}}<\infty$, there exists $0 \neq w \in G_{\frac{3}{2}} V$ such that $L_{-1} w=0$. 
Suppose $w=G_{\frac{3}{2}} u$ for some $u \in V$. For all $l \geq k>\frac{3}{2}$, using (1) we have

$$
E_{l} w=E_{l} G_{\frac{3}{2}} u=\left\{\begin{aligned}
-G_{\frac{3}{2}} E_{l} u+\left[E_{l}, G_{\frac{3}{2}}\right] u=0, & \text { if } k \in \frac{1}{2}+\mathbb{Z}_{+}, \\
G_{\frac{3}{2}} E_{l} u+\left[E_{l}, G_{\frac{3}{2}}\right] u=0, & \text { if } k \in \mathbb{Z}_{+} .
\end{aligned}\right.
$$

Hence

$$
E_{l} w=0, \forall l=-1, k, k+\frac{1}{2}, k+1 . \cdots
$$

Since $\left[L_{-1}, E_{l}\right] \neq 0$ for all $l \geq \frac{3}{2}$, inductively, we get

$$
E_{l} w=0, \forall l=\frac{1}{2}, 1, \frac{3}{2}, 2, \cdots .
$$

Hence $M$ is a Harish-Chandra module by the Principal Fact. A contradiction. Then the lemma follows.

By Lemma 1, we see that there exist at most two elements in $\left\{\lambda+k \mid k \in \frac{\mathbb{Z}}{2}\right\}$ which correspond to finite-dimensional weight spaces or trivial vector spaces in $M$. Moreover, if there are two, then they are adjacent.

Lemma 2. (i) Let $0 \neq v \in M$ be such that $G_{\frac{1}{2}} v=0$. Then

$$
\left(\frac{1}{2} L_{1} G_{\frac{1}{2}}-G_{\frac{3}{2}}\right) G_{\frac{3}{2}} v=0 .
$$

(ii) Let $0 \neq w \in M$ be such that $G_{-\frac{1}{2}} w=0$. Then

$$
\left(\frac{1}{2} L_{-1} G_{-\frac{1}{2}}+G_{-\frac{3}{2}}\right) G_{-\frac{3}{2}} w=0
$$

Proof. Note that $L_{1} v=G_{\frac{1}{2}} G_{\frac{1}{2}} v=0, L_{-1} w=G_{-\frac{1}{2}} G_{-\frac{1}{2}} w=0$, we can easily check by a direct calculation that Lemma 2 holds.

Lemma 3. Let $M$ be a simple weight $N S$-module satisfying $\operatorname{dim} M_{\mu}<\infty$ and $\operatorname{dim} M_{\mu+\frac{1}{2}}<\infty$. Then $\mu \in\left\{-1, \frac{1}{2}\right\}$.

Proof. Let $V$ be the kernel of $G_{\frac{1}{2}}: M_{\mu-\frac{1}{2}} \rightarrow M_{\mu}$. Since $\operatorname{dim} M_{\mu-\frac{1}{2}}=\infty$ and $\operatorname{dim} M_{\mu}<\infty$, we see that $\operatorname{dim} V=\infty$. For any $0 \neq v \in V$, consider the element $G_{\frac{3}{2}} v$. By the Principal Fact, $G_{\frac{3}{2}} v=0$ would imply that $M$ is a Harish-Chandra module, a contradiction. Hence $G_{\frac{3}{2}} v \neq 0$, in particular, $\operatorname{dim} G_{\frac{3}{2}} V=\infty$. This implies that there exists $w \in G_{\frac{3}{2}} V$ such that $w \neq 0$ and $G_{-\frac{1}{2}} w=0$ since $\operatorname{dim} M_{\mu+\frac{1}{2}}<\infty$. From Lemma 2 we have $\left(\frac{1}{2} L_{1} G_{\frac{1}{2}}-G_{\frac{3}{2}}\right) w=0$. In particular, we have $L_{-1} G_{-\frac{1}{2}}\left(\frac{1}{2} L_{1} G_{\frac{1}{2}}-G_{\frac{3}{2}}\right) w=0$. By a direct calculation we obtain

$$
L_{-1} G_{-\frac{1}{2}}\left(\frac{1}{2} L_{1} G_{\frac{1}{2}}-G_{\frac{3}{2}}\right) \equiv 2 L_{0}^{2}-3 L_{0} \bmod U(N S) G_{-\frac{1}{2}}
$$


But $w \in M_{\mu+1}$, which means $L_{0} w=(\mu+1) w$ and hence

$$
2(\mu+1)^{2}-3(\mu+1)=0 .
$$

So $\mu \in\left\{-1, \frac{1}{2}\right\}$.

From Lemma 3 we know that if $M$ has two finite dimension weight spaces, then they must be $M_{-1}, M_{-\frac{1}{2}}$ or $M_{\frac{1}{2}}, M_{1}$.

Lemma 4. $\mu \neq-1$ and $\mu \neq \frac{1}{2}$.

Proof. By the symmetry, we need only to prove that $\mu \neq \frac{1}{2}$. Let $V$ be the kernel of $G_{\frac{1}{2}}: M_{0} \rightarrow M_{\frac{1}{2}}$. Then $\operatorname{dim} V=\infty$. For $v \in V$, using $G_{\frac{1}{2}} v=L_{0} v=0$, we have

$$
G_{\frac{1}{2}} G_{-\frac{1}{2}} v=2 L_{0} v-G_{-\frac{1}{2}} G_{\frac{1}{2}} v=0 .
$$

If $G_{-\frac{1}{2}} V$ were infinite-dimensional, there would exists $0 \neq w \in G_{-\frac{1}{2}} V$ such that $G_{\frac{1}{2}} w=0$ (by $(2)$ ) and $G_{\frac{3}{2}} w=0$ (since $\operatorname{dim} M_{1}<\infty$ ). Then the Principal Fact would then imply that $M$ is a Harish-Chandra module, a contradiction. Hence

$$
\operatorname{dim} G_{-\frac{1}{2}} V<\infty
$$

This means that the kernel $W$ of the linear map

$$
G_{-\frac{1}{2}}: V \rightarrow M_{-\frac{1}{2}}
$$

is infinite-dimensional. For every $x \in W$ we have

$$
G_{\frac{1}{2}} G_{-\frac{3}{2}} x=2 L_{-1} x-G_{-\frac{3}{2}} G_{\frac{1}{2}} x=0 .
$$

If there exists $0 \neq x \in W$ such that $G_{-\frac{3}{2}} x=0$, then we would have $G_{-\frac{3}{2}} x=$ $G_{-\frac{1}{2}} x=0$ and the Principal Fact would imply that $M$ is a Harish-Chandra module, a contradiction. Thus

$$
\operatorname{dim} G_{-\frac{3}{2}} W=\infty
$$

Let $H$ denote the kernel of the linear map $L_{2}: G_{-\frac{3}{2}} W \rightarrow M_{\frac{1}{2}}$. Since $\operatorname{dim} G_{-\frac{3}{2}} W=\infty$ and $\operatorname{dim} M_{\frac{1}{2}}<\infty$, we have $\operatorname{dim} H=\infty$. For every $y \in H$, we also have $G_{\frac{1}{2}} y=0$ by (3), implying by induction that $E_{k} H=0$ for all $k=\frac{1}{2}, 1,2, \frac{5}{2}, 3, \frac{7}{2}, 4, \cdots$. If $G_{\frac{3}{2}} h=0$ for some $0 \neq h \in H$, then the Principal Fact implies that $M$ is a Harish-Chandra module, a contradiction. Hence

$$
\operatorname{dim} G_{\frac{3}{2}} H=\infty
$$

For every $h \in H$ and $k \geq 2$, we have

$$
E_{k} G_{\frac{3}{2}} h= \begin{cases}2 L_{\frac{3}{2}+k} h-G_{\frac{3}{2}} G_{k} h=0, & \text { if } k \in \frac{1}{2}+\mathbb{Z}_{+}, \\ \left(\frac{3}{2}-\frac{k}{2}\right) G_{\frac{3}{2}+k} h+G_{\frac{3}{2}} L_{k} h=0, & \text { if } k \in \mathbb{Z}_{+} .\end{cases}
$$


Hence

$$
E_{k} G_{\frac{3}{2}} h=0, \forall k=2, \frac{5}{2}, 3, \frac{7}{2}, 4, \frac{9}{2}, \cdots
$$

Let, finally, $K$ denote the infinite-dimensional kernel of the linear map

$$
G_{\frac{1}{2}}: G_{\frac{3}{2}} H \rightarrow M_{\frac{1}{2}}
$$

If $G_{\frac{3}{2}} z=0$ for some $0 \neq z \in K$, then the Principal Fact implies that $M$ is a HarishChandra module, a contradiction. Hence $G_{\frac{3}{2}} z \neq 0, \forall 0 \neq z \in K$. For every $z \in K$ and $k \geq 2$, by (4), we have $\operatorname{dim} G_{\frac{3}{2}} K=\infty$ and

$$
E_{k} G_{\frac{3}{2}} z= \begin{cases}2 L_{\frac{3}{2}+k} z-G_{\frac{3}{2}} G_{k} z=0, & \text { if } k \in \frac{1}{2}+\mathbb{Z}_{+}, \\ \left(\frac{3}{2}-\frac{k}{2}\right) G_{\frac{3}{2}+k} z+G_{\frac{3}{2}} L_{k} z=0 . & \text { if } k \in \mathbb{Z}_{+} .\end{cases}
$$

Hence $E_{k} G_{\frac{3}{2}} K=0$ for all $k \geq 2$. At the same time, since $\operatorname{dim} G_{\frac{3}{2}} K=\infty$ and $\operatorname{dim} M_{1}<\infty$, we can choose some $0 \neq t \in G_{\frac{3}{2}} K$ such that $G_{-\frac{1}{2}} t=0$, by induction, we get $E_{i} t=0$ for all $i>0$ and thus $M$ is a Harish-Chandra module by the Principal Fact. This last contradiction completes the proof of lemma 4.

By lemma 1, Lemma 3 and Lemma 4, we get the following lemma immediately:

Lemma 5. There is at most one element $\mu \in\left\{\lambda+k \mid k \in \frac{\mathbb{Z}}{2}\right\}$ such that $\operatorname{dim} M_{\mu}<\infty$.

Now the proof of Theorem 1 follows from the following Lemma:

Lemma 6. There is no $\mu \in\left\{\lambda+k \mid k \in \frac{\mathbb{Z}}{2}\right\}$ such that $\operatorname{dim} M_{\mu}<\infty$.

Proof. Suppose that $\operatorname{dim} M_{\mu}<\infty$ and $\operatorname{dim} M_{\nu}=\infty$ for all $\mu \neq \nu \in\left\{\lambda+k \mid k \in \frac{\mathbb{Z}}{2}\right\}$. Define

$$
\begin{aligned}
& V=\operatorname{ker}\left(G_{\frac{1}{2}}: M_{\mu-\frac{1}{2}} \rightarrow M_{\mu}\right) \cap \operatorname{ker}\left(G_{\frac{1}{2}} G_{-\frac{3}{2}} G_{\frac{3}{2}}: M_{\mu-\frac{1}{2}} \rightarrow M_{\mu}\right) \cap \operatorname{ker}\left(L_{-1} G_{\frac{3}{2}}: M_{\mu-\frac{1}{2}} \rightarrow M_{\mu}\right), \\
& W=\operatorname{ker}\left(G_{-\frac{1}{2}}: M_{\mu+\frac{1}{2}} \rightarrow M_{\mu}\right) \cap \operatorname{ker}\left(G_{-\frac{1}{2}} G_{\frac{3}{2}} G_{-\frac{3}{2}}: M_{\mu-\frac{1}{2}} \rightarrow M_{\mu}\right) \cap \operatorname{ker}\left(L_{1} G_{-\frac{3}{2}}: M_{\mu-\frac{1}{2}} \rightarrow M_{\mu}\right) .
\end{aligned}
$$

Since $\operatorname{dim} M_{\mu-\frac{1}{2}}=\infty$ and $\operatorname{dim} M_{\mu}<\infty, V$ is a vector subspace of finite codimension in $M_{\mu-\frac{1}{2}}$. Since $\operatorname{dim} M_{\mu+\frac{1}{2}}=\infty$ and $\operatorname{dim} M_{\mu}<\infty, W$ is a vector subspace of finite codimension in $M_{\mu+\frac{1}{2}}$. In order not to get a direct contradiction using the Principal Fact, we assume that $G_{\frac{3}{2}} v \neq 0$ for all $0 \neq v \in V$ and $G_{-\frac{3}{2}} w \neq 0$ for all $0 \neq w \in W$. Then $\operatorname{dim} G_{\frac{3}{2}} V=\infty$ and $\operatorname{dim} G_{-\frac{3}{2}} W=\infty$.

Claim $\mu \neq \pm 1$. Moreover, for any $v \in V, w \in W$, we have

$$
\begin{aligned}
G_{\frac{1}{2}} G_{-\frac{1}{2}} G_{\frac{3}{2}} v & =\tau G_{\frac{3}{2}} v, \\
G_{-\frac{1}{2}} G_{\frac{1}{2}} G_{-\frac{3}{2}} w & =\tau^{\prime} G_{-\frac{3}{2}} w,
\end{aligned}
$$

where $\tau=\frac{(\mu+1)(2 \mu-1)}{\mu-1}$ and $\tau^{\prime}=\frac{(\mu-1)(2 \mu+1)}{\mu+1}$. 
Proof of the Claim. It can be checked directly that

$$
L_{-1} G_{-\frac{1}{2}}\left(\frac{1}{2} L_{1} G_{\frac{1}{2}}-G_{\frac{3}{2}}\right) \equiv 2 L_{0}^{2}-3 L_{0}-L_{0} G_{\frac{1}{2}} G_{-\frac{1}{2}}+2 G_{\frac{1}{2}} G_{-\frac{1}{2}} \bmod U(N S) L_{-1},
$$

and

$$
L_{1} G_{\frac{1}{2}}\left(\frac{1}{2} L_{-1} G_{-\frac{1}{2}}+G_{-\frac{3}{2}}\right) \equiv-2 L_{0}^{2}-3 L_{0}+L_{0} G_{-\frac{1}{2}} G_{\frac{1}{2}}+2 G_{-\frac{1}{2}} G_{\frac{1}{2}} \bmod U(N S) L_{1} .
$$

For any $0 \neq v \in V$, by Lemma 2(i) and (7), we have

$$
2 L_{0}^{2} G_{\frac{3}{2}} v-3 L_{0} G_{\frac{3}{2}} v-L_{0} G_{\frac{1}{2}} G_{-\frac{1}{2}} G_{\frac{3}{2}} v+2 G_{\frac{1}{2}} G_{-\frac{1}{2}} G_{\frac{3}{2}} v=0 .
$$

Then

$$
\left(2(\mu+1)^{2}-3(\mu+1)\right) G_{\frac{3}{2}} v-(\mu-1) G_{\frac{1}{2}} G_{-\frac{1}{2}} G_{\frac{3}{2}} v=0 .
$$

If $\mu=1$, then $G_{\frac{3}{2}} v=0$, a contradiction. Thus $\mu \neq 1$, and (4) holds.

For any $0 \neq w \in W$, by Lemma 2(ii) and (8), we have

$$
-2 L_{0}^{2} G_{-\frac{3}{2}} w-3 L_{0} G_{-\frac{3}{2}} w+L_{0} G_{-\frac{1}{2}} G_{\frac{1}{2}} G_{-\frac{3}{2}} w+2 G_{-\frac{1}{2}} G_{\frac{1}{2}} G_{-\frac{3}{2}} w=0 .
$$

Then

$$
\left(2(\mu-1)^{2}+3(\mu-1)\right) G_{-\frac{3}{2}} w-(\mu+1) G_{-\frac{1}{2}} G_{\frac{1}{2}} G_{-\frac{3}{2}} w=0 .
$$

If $\mu=-1$, then $G_{-\frac{3}{2}} w=0$, a contradiction. Thus $\mu \neq-1$, and (6) holds. This completes the proof of the Claim.

By (4) and (6), we see that $G_{\frac{1}{2}} G_{-\frac{1}{2}} G_{\frac{3}{2}} v=0$ if and only if $\mu=\frac{1}{2}, G_{-\frac{1}{2}} G_{\frac{1}{2}} G_{-\frac{3}{2}} w=$ 0 if and only if $\mu=-\frac{1}{2}$. There only two cases may occur: $\mu \notin\left\{1,-1, \frac{1}{2}\right\}$ or $\mu \notin\left\{1,-1,-\frac{1}{2}\right\}$. Because of the symmetry of our situation, to complete the proof of the Theorem, it is enough to show that a contradiction can be derived when $\mu \notin\left\{1,-1, \frac{1}{2}\right\}$.

Suppose $\mu \notin\left\{1,-1, \frac{1}{2}\right\}$, then $\tau \neq 0$ and $G_{-\frac{1}{2}} G_{\frac{3}{2}} v \neq 0$ for any $0 \neq v \in V$. Set

$$
S=G_{-\frac{1}{2}} G_{\frac{3}{2}} V \cap W
$$

Since both $G_{-\frac{1}{2}} G_{\frac{3}{2}} V$ and $W$ have finite codimension in $M_{\mu+\frac{1}{2}}$, we have $\operatorname{dim} S=\infty$.

Note that

$$
G_{-\frac{3}{2}}\left(\frac{1}{2} L_{1} G_{\frac{1}{2}}-G_{\frac{3}{2}}\right) \equiv-G_{\frac{1}{2}} G_{-\frac{1}{2}}-\frac{1}{2} L_{1} G_{\frac{1}{2}} G_{-\frac{3}{2}}+G_{\frac{3}{2}} G_{-\frac{3}{2}}-\frac{2}{3} c \bmod U(N S) L_{-1} .
$$

For any $v \in V$, by Lemma 2(i), (4) and the definition of $V$, we have

$$
G_{\frac{3}{2}} G_{-\frac{3}{2}} G_{\frac{3}{2}} v=G_{\frac{1}{2}} G_{-\frac{1}{2}} G_{\frac{3}{2}} v+\frac{2}{3} c G_{\frac{3}{2}} v=p G_{\frac{3}{2}} v \text {, for some } p \in \mathbb{C} \text {. }
$$

Similarly, For any $w \in W$, by

$$
G_{\frac{3}{2}}\left(\frac{1}{2} L_{-1} G_{-\frac{1}{2}}+G_{-\frac{3}{2}}\right) \equiv G_{-\frac{1}{2}} G_{\frac{1}{2}}-\frac{1}{2} L_{-1} G_{-\frac{1}{2}} G_{\frac{3}{2}}-G_{-\frac{3}{2}} G_{\frac{3}{2}}+\frac{2}{3} c \bmod U(N S) L_{1},
$$


Lemma 2(ii) , (6) and the definition of $W$, we have

$$
G_{-\frac{3}{2}} G_{\frac{3}{2}} G_{-\frac{3}{2}} w=G_{-\frac{1}{2}} G_{\frac{1}{2}} G_{-\frac{3}{2}} w+\frac{2}{3} c G_{-\frac{3}{2}} w=q G_{-\frac{3}{2}} w, \text { for some } q \in \mathbb{C} \text {. }
$$

Choose $v \in V$ such that $0 \neq G_{-\frac{1}{2}} G_{\frac{3}{2}} v \in S$. Set

$$
x=G_{\frac{3}{2}} v, y=G_{-\frac{1}{2}} x, h=G_{-\frac{3}{2}} x, z=G_{-\frac{3}{2}} y .
$$

By the definitions of $W, S$ and (10), we have

$$
G_{-\frac{1}{2}}\left(G_{\frac{3}{2}} G_{-\frac{3}{2}} y-q y\right)=0-0=0
$$

and

$$
G_{-\frac{3}{2}}\left(G_{\frac{3}{2}} G_{-\frac{3}{2}} y-q y\right)=G_{-\frac{3}{2}} G_{\frac{3}{2}} G_{-\frac{3}{2}} y-q G_{-\frac{3}{2}} y=0 .
$$

So $G_{\frac{3}{2}} G_{-\frac{3}{2}} y-q y=0$ by the Principal Fact, and we get the formula:

$$
G_{\frac{3}{2}} z=q y
$$

Moreover, we have

$G_{\frac{1}{2}} z=G_{\frac{1}{2}} G_{-\frac{3}{2}} y=\left(2 L_{-1}-G_{-\frac{3}{2}} G_{\frac{1}{2}}\right) y=2 G_{-\frac{1}{2}}^{2} y-G_{-\frac{3}{2}} G_{\frac{1}{2}} G_{-\frac{1}{2}} G_{\frac{3}{2}} v=-\tau G_{-\frac{3}{2}} x=-\tau h$ i.e.,

$$
G_{\frac{1}{2}} z=-\tau h
$$

Let $U_{+}$and $U_{-}$denote the subalgebras of $U(N S)$, generated by $G_{\frac{1}{2}}, G_{\frac{3}{2}}$ and $G_{-\frac{1}{2}}, G_{-\frac{3}{2}}$, respectively. We want to prove that the following vector space

$$
N=U_{+} x \oplus U_{+} y \oplus U_{-} z \oplus U_{-} h
$$

is a proper weight submodule of $M$, which will derive a contradiction, as desired.

Since $x, y, z$ and $h$ are all eigenvectors for $L_{0}$, we see that $N$ decomposes into a direct sum of weight spaces which are obviously finite-dimensional. It remains to show that $N$ is stable under the action of the following four operators: $G_{\frac{1}{2}}, G_{\frac{3}{2}}, G_{-\frac{1}{2}}$ and $G_{-\frac{3}{2}}$. That $G_{\frac{i}{2}} U_{+} x \subset U_{+} x, G_{\frac{i}{2}} U_{+} y \subset U_{+} y, G_{-\frac{i}{2}} U_{-} h \subset U_{-} h$ and $G_{-\frac{i}{2}} U_{-} z \stackrel{\frac{1}{2}}{\complement}$ $U_{-} z$ is clear for $i=1,3$.

For any $a \in U_{+}, a^{\prime} \in U_{-}$, there exists $a_{i, j}, b_{i, j}, c_{i, j} \in U_{+}$and $a_{i, j}^{\prime}, b_{i, j}^{\prime}, c_{i, j}^{\prime} \in U_{-}$ such that

$$
\begin{gathered}
G_{-\frac{1}{2}} a=a G_{-\frac{1}{2}}+\sum_{i, j} a_{i, j} L_{0}^{i} c^{j} \\
G_{-\frac{3}{2}} a=a G_{-\frac{3}{2}}+\sum_{i, j} a_{i, j} L_{0}^{i} c^{j}+\sum_{i, j} b_{i, j} L_{0}^{i} c^{j} L_{-1}+\sum_{i, j} c_{i, j} L_{0}^{i} c^{j} G_{-\frac{1}{2}} \\
G_{\frac{1}{2}} a^{\prime}=a^{\prime} G_{\frac{1}{2}}+\sum_{i, j} a_{i, j}^{\prime} L_{0}^{i} c^{j}
\end{gathered}
$$




$$
G_{\frac{3}{2}} a^{\prime}=a^{\prime} G_{\frac{3}{2}}+\sum_{i, j} a_{i, j}^{\prime} L_{0}^{i} c^{j}+\sum_{i, j} b_{i, j}^{\prime} L_{0}^{i} c^{j} L_{1}+\sum_{i, j} c_{i, j}^{\prime} L_{0}^{i} c^{j} G_{\frac{1}{2}} .
$$

Thus, to show $G_{-\frac{i}{2}} U_{+} x, G_{-\frac{i}{2}} U_{+} y, G_{\frac{i}{2}} U_{-} h, G_{\frac{i}{2}} U_{-} z \subset N$, we need only to show that $G_{-\frac{i}{2}} x, G_{-\frac{i}{2}} y, G_{\frac{i}{2}} h, G_{\frac{i}{2}} z, G_{-\frac{i}{2}} G_{\frac{j}{2}} x, G_{-\frac{i}{2}} G_{\frac{j}{2}} y, G_{\frac{i}{2}} G_{-\frac{j}{2}} h, G_{\frac{i}{2}} G_{-\frac{j}{2}} z \in N$ for $i, j=1,3$.

Now we can check the following formulas one by one via the definitions of $V, W, S$ and (5)-(13):

$$
\begin{aligned}
& G_{-\frac{1}{2}} x=y, \\
& G_{-\frac{1}{2}} y=0 \text {, } \\
& G_{-\frac{3}{2}} x=h \text {, } \\
& G_{-\frac{3}{2}} y=z, \\
& G_{\frac{1}{2}} h=G_{\frac{1}{2}} G_{-\frac{3}{2}} G_{\frac{3}{2}} v=0, \\
& G_{\frac{1}{2}} z=-\tau h, \\
& G_{\frac{3}{2}} h=G_{\frac{3}{2}} G_{-\frac{3}{2}} G_{\frac{3}{2}} v=p G_{\frac{3}{2}} v=p x, \\
& G_{\frac{3}{2}} z=q y, \\
& G_{\frac{1}{2}} G_{-\frac{3}{2}} x=0 \text {, } \\
& G_{\frac{3}{2}} G_{-\frac{3}{2}} x=p x, \\
& G_{\frac{1}{2}} G_{-\frac{3}{2}} y=-\tau h, \\
& G_{\frac{3}{2}} G_{-\frac{3}{2}} y=q y, \\
& G_{\frac{1}{2}} G_{\frac{1}{2}} G_{-\frac{3}{2}} y=0, \\
& G_{\frac{3}{2}} G_{\frac{1}{2}} G_{-\frac{3}{2}} y=-\tau p x \\
& L_{-1} x=G_{-\frac{1}{2}}^{2} x=0, \\
& L_{-1} y=G_{-\frac{1}{2}}^{2} y=0 \text {, } \\
& G_{-\frac{1}{2}} G_{\frac{3}{2}} h=p y, \\
& G_{-\frac{3}{2}} G_{\frac{3}{2}} h=p h, \\
& G_{-\frac{1}{2}} G_{\frac{3}{2}} z=0 \text {, } \\
& G_{-\frac{3}{2}} G_{\frac{3}{2}} z=q z, \\
& G_{-\frac{1}{2}} G_{-\frac{1}{2}} G_{\frac{3}{2}} h=0, \\
& G_{-\frac{3}{2}} G_{-\frac{1}{2}} G_{\frac{3}{2}} h=p z, \\
& L_{1} h=G_{\frac{1}{2}}^{2} h=0, \\
& L_{1} z=G_{\frac{1}{2}}^{2} z=0 .
\end{aligned}
$$

This completes the proof of Lemma 6 and then of Theorem 1. 


\section{References}

[1] Goddard, P., Kent, A. and Olive, D. Unitary representations of the Virasoro and super-Virasoro algebras. Commun. Math. Phys. 103(1986): 105-119.

[2] Iohara, K., Koga, Y. Representation theory of Neveu-Schwarz and Remond algebras I: Verma modules. Adv. Math. 177(2003): 61-69.

[3] Iohara, K., Koga, Y. Representation theory of Neveu-Schwarz and Remond algebras II: Fock modules. Ann. Inst. Fourier 53(2003): 1755C1818.

[4] Kac, V. G.(1982). Some problems of infinite-dimensional Lie algebras and their representations. lecture notes in math. 933, Springer, New York, pp. 117-126.

[5] Mathieu, O. Classification of Harish-Chandra modules over the Virasoro Lie algebra. Invent. Math. 107(1992): 225-234.

[6] Mazorchuk, V. Zhao, K., Classification of simple weight Virasoro modules with a finite-dimensional weight space. J. Algebra, 307(2007): 209-214.

[7] Su, Y. Classification of Harish-Chandra modules over the super-Virasoro algebras. Comm. Algegra 23(1995): 3653-3675.

Note This artical has been accepted by Communications in algebra in $24 / 03 / 2011$. 